\title{
On Generalizations of Extending Modules
}

\author{
FATIH KARABACAK \\ Anadolu University, Education Faculty, Department of Mathematics, 26470, Es- \\ kisehir, Turkey \\ e-mail : fatihkarabacak@anadolu.edu.tr
}

Abstract. A module $M$ is said to be SIP-extending if the intersection of every pair of direct summands is essential in a direct summand of $M$. SIP-extending modules are a proper generalization of both SIP-modules and extending modules. Every direct summand of an SIP-module is an SIP-module just as a direct summand of an extending module is extending. While it is known that a direct sum of SIP-extending modules is not necessarily SIP-extending, the question about direct summands of an SIP-extending module to be SIP-extending remains open. In this study, we show that a direct summand of an SIP-extending module inherits this property under some conditions. Some related results are included about $C_{11}$ and SIP-modules.

\section{Introduction}

Throughout this paper all rings are associative with unity and $R$ always denotes such a ring. Modules are unital and for an abelian group $M$, we use $M_{R}\left(\operatorname{resp} .{ }_{R} M\right)$ to denote a right (resp. left) $R$-module. Let $M$ be a $R$-module and $N$ a submodule of $M$. We use $N \leq_{e} M$ and $N \leq_{d} M$ to denote that $N$ is essential in $M$ and $N$ is a direct summand of $M$, respectively. Moreover we use $\operatorname{End}\left(M_{R}\right)$ and $r(m)$ to denote the ring of endomorphisms of $M$ and the right annihilator in $R$ of an element $m$ in $M$, i.e., $r(m)=\{r \in R: m . r=0\}$. Recall that a ring is called Abelian if every idempotent is central. For any unexplained terminology please see [1] and [5].

A module $M_{R}$ has the Summand Intersection Property, SIP, if the intersection of every pair of direct summands of $M_{R}$ is a direct summand of $M_{R}$. The study of modules having SIP was motivated by the following result of Kaplansky [7]: every free module over any principal ideal domain has SIP. The Summand Intersection Property has been studied by many authors (see e.g. [2], [3], [6], [8] and [17].)

Recall that a module $M$ is called an extending module (or a CS-module) if every submodule is essential in a direct summand of $M$. In [5] and [11], extending modules were studied in detail.

The concept of $C_{11}$-modules was introduced in [15] as a generalization of extending modules. A module $M$ is called $C_{11}$-module (or satisfies $C_{11}$ )[15] if every

Received August 3, 2008; accepted November 4, 2008.

2000 Mathematics Subject Classification: 16D10, 16D15.

Key words and phrases: SIP-extending modules, summand intersection property, extending modules. 
submodule of $M$ has a complement which is a direct summand. It is known that a direct summand of a $C_{11}$-module is not a $C_{11}$-module, in general (see [16, Exercise 4]). A module is called a $C_{11}^{+}$-module if its every direct summand is a $C_{11}$-module [15]. In this paper we further the study of SIP-extending modules and we show that if $M$ is a $C_{11}$-module which is also SIP-extending then every direct summand of $M$ is a $C_{11}$-module, i.e., $M$ is $C_{11}^{+}$-module (see Proposition 7 ). In the main result we show that if $M$ is an SIP-extending module such that $\operatorname{End}\left(M_{R}\right)$ is Abelian then every direct summand of $M$ is SIP-extending.

\section{SIP-extending modules}

In [9], a module $M$ is called an SIP-extending module provided that the intersection of every pair of direct summands of $M$ is essential in a direct summand of $M$. We say a ring $R$ is a right $S I P$-extending ring if the module $R_{R}$ is an SIP-extending module, i.e., for every pair of idempotents $e, c$ in $R$ there exists $g^{2}=g \in R$ such that $e R \cap c R$ is essential in $g R$. Examples of SIP-extending modules include every extending (hence every injective) module, every uniform module, every semisimple module and every module having the SIP (e.g. any Baer module [13]). The concept of an SIP-extending module is a proper generalization of both SIP-modules and extending modules, as shown by the following examples.

Example 1. Let $F$ be any field and $V$ be a $F$-vector space with $\operatorname{dim} V_{F} \geq 2$. Let

$$
R=\left\{\left[\begin{array}{ll}
a & v \\
0 & a
\end{array}\right]: a \in F, v \in V\right\},
$$

be the trivial extension of $F$ by $V$. Then $R$ is a right SIP-extending ring however since $\operatorname{dim} V_{F} \geq 2, R$ is not right extending ring.

Example 2([4, Exercise 1.5]). Let $F$ be a field and

$$
T=\left\{\left[\begin{array}{cccc}
a & x & 0 & 0 \\
0 & b & 0 & 0 \\
0 & 0 & b & y \\
0 & 0 & 0 & a
\end{array}\right]: a, b, x, y \in F\right\}
$$

Let

$$
e=e^{2}=\left[\begin{array}{llll}
0 & 1 & 0 & 0 \\
0 & 1 & 0 & 0 \\
0 & 0 & 1 & 0 \\
0 & 0 & 0 & 0
\end{array}\right]
$$

and

$$
c=c^{2}=\left[\begin{array}{llll}
0 & 0 & 0 & 0 \\
0 & 1 & 0 & 0 \\
0 & 0 & 1 & 0 \\
0 & 0 & 0 & 0
\end{array}\right]
$$


Then $e T \cap c T$ is nilpotent. Hence $e T \cap c T$ is not a direct summand of $T$. It follows that $T$ does not have SIP. However it is a right SIP-extending ring.

It is well known that every direct summand of SIP-modules is an SIP-module and every direct summand of extending modules is an extending module. This result led us to the following question.

Question: Let $M$ be an SIP-extending module and $N$ be a direct summand of $M$. Is $N$ an SIP-extending module?

In [9] we have provided a positive answer to the direct summand question under the condition that the summand is the unique closure of each of its essential submodules.

Proposition 3([9, Lemma 6]). Let $M$ be an SIP-extending module, and let $N$ be a direct summand of $M$. Suppose that $N$ is the unique closure in $M$ of any of its essential submodules. Then $N$ is also an SIP-extending module.

Definition 4([14]). Let $M$ be a module. If every submodule has a unique closure in $M$ then $M$ is called UC-module.

Proposition 5. Let $M$ be a UC-module. Then $M$ has SIP if and only if $M$ is SIP-extending.

Proof. It is clear that if $M$ has SIP then $M$ is SIP-extending. Conversely, let $S_{1}$ and $S_{2}$ be direct summands of $M$. Then by hypothesis $S_{1} \cap S_{2} \leq_{e} P$ for some $P \leq_{d} M$. By the main theorem in [14], intersection of two closed submodules is closed hence $S_{1} \cap S_{2}=P$. Thus $M$ has SIP.

The following lemma is proved in [9, Theorem 8].

Lemma 6. Let $M$ be a $C_{11}$-module and $E$ be a submodule of $M$. If for every direct summand $D$ of $M, E \cap D$ is essential in a direct summand of $E$ then $E$ is a $C_{11}$-module.

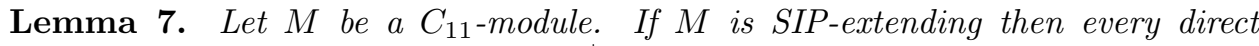
summand of $M$ is $C_{11}$ (i.e., $M$ has $C_{11}^{+}$).

Proof. By Lemma 6 and the definition of SIP-extending.

Recall that $R$ is said to Abelian if every idempotent of $R$ is central. Note that every finite dimension module has an Abelian endomorphism ring by [11]. We have the following result for SIP-extending Abelian rings.

Proposition 8. Let $R$ be an Abelian ring then

i) $R$ is SIP-extending $(S I P)$ if and only if $R[x]$ is SIP-extending $(S I P)$.

ii) $R$ is SIP-extending $(S I P)$ if and only if $R[[x]]$ is SIP-extending $(S I P)$.

Proof. Since $R$ is an Abelian ring, the result follows by [10, Lemma 8].

Next, we provide an answer to the direct summand question for an SIPextending module under another special condition. The result shows that a fully 
invariant direct summand of an SIP-extending module inherits the property. It also completes the sufficiency part of $[9$, Theorem 11] in which only the necessity was established.

Theorem 9. Let $M=\oplus_{i \in I} M_{i}$ be a direct sum of fully invariant submodules $M_{i}$ of $M$ where $I$ is an index set. Then $M$ is an SIP-extending module if and only if $M_{i}$, $\forall i \in I$ is an SIP-extending module.

Proof. Let $M$ be an SIP-extending module and $M_{i}$ be a fully invariant direct summand of $M$. If $L$ and $K$ are direct summand of $M_{i}$ then there exist $P \leq_{d} M$ ( $M=P \oplus Q$, for some $Q \leq M)$ such that $L \cap K \leq_{e} P$. Since $M_{i}$ is a fully invariant direct summand of $M$ and $M=P \oplus Q$ then $M_{i}=\left(M_{i} \cap P\right) \oplus\left(M_{i} \cap Q\right)$. Therefore $L \cap K \leq_{e} M_{i} \cap P \leq_{d} M_{i}$. So $M_{i}$ is an SIP-extending module. Converse follows from $[9$, Theorem 11]. We include a brief outline for the convenience of the reader. Let $S$ be any direct summand of $M$. So $S=\oplus\left(S \cap M_{i}\right)$. Now let $S, T$ be direct summands of $M$. Hence, $S \cap T=\oplus\left[\left(S \cap M_{i}\right) \cap\left(T \cap M_{i}\right)\right]$. Therefore, there exists a direct summand $K_{i}$ of $M_{i}$ which contains $\left(S \cap M_{i}\right) \cap\left(T \cap M_{i}\right)$ as an essential submodule.

Corollary 10. $M_{R}$ is an SIP-extending module such that End $\left(M_{R}\right)$ is Abelian. Then every direct summand of $M$ is SIP-extending.

Proof. Let $M$ be an SIP-extending module and $M_{1}$ be a direct summand of $M$. Since $\operatorname{End}\left(M_{R}\right)$ is Abelian $M_{1}$ is a fully invariant submodule of $M$. By Theorem $9, M_{1}$ is an SIP-extending module.

Definition 11. Let $M$ be a $R$-module. $M$ is said to be multiplication module if for each $X \leq M$ there exists $A_{R} \leq R_{R}$ such that $X=M A$

Corollary 12. Let $M$ be an SIP-extending module, then any direct summand of $M$ is SIP-extending if $M$ satisfies any of the following conditions.

(i) $M_{R}=R_{R}$ and $R$ is Abelian.

(ii) $M$ is a multiplication module and $R$ is commutative.

Proof. (i) Immediate by Corollary 10.

(ii) Assume that $M$ is multiplication module and $R$ is commutative. Note that every submodule of a multiplication module is fully invariant. Now Theorem 9 yields the result.

Recall that a module $M$ satisfies the $C_{3}$ condition whenever $K, L$ are direct summand of $M$ with $K \cap L=0$ then $K \oplus L \leq_{d} M$ (see [11]). Note that the $\mathbb{Z}_{\text {- }}$ module $(\mathbb{Z} \oplus \mathbb{Z})$ is an SIP-extending which does not satisfy the $C_{3}$ condition (see, for example [3]). Now we provide an example which shows that a module satisfying the $C_{3}$ property does not have to be SIP-extending either.

Example 13. Let $F$ be a field and $R=\left(\begin{array}{cc}F & F \\ 0 & F\end{array}\right)$ be the ring of upper triangular matrices over $F, N=\left(\begin{array}{cc}0 & F \\ 0 & F\end{array}\right)$ and $L=\left(\begin{array}{cc}F & F \\ 0 & 0\end{array}\right)$ left ideals of $R$ and $M=$ 
$R / L$. Let $U=N \oplus M$. Then ${ }_{R} U$ satisfies the $C_{3}$ condition and is a UC-module but does not have the SIP as a left R-module. By Proposition 5, U is not SIP-extending as a left R-module.

We conclude this paper with some results for $C_{11}$-modules. Recall that a module $M$ is said to satisfy the full (finite) exchange property if for any module $G$ and any two direct sum decompositions $G=M^{\prime} \oplus N=\oplus_{i \in I} A_{i}$ where $M^{\prime} \cong M$ and $I$ is any (finite) index set, there are submodules $B_{i}$ of $A_{i}, i \in I$, such that $G=M^{\prime} \oplus\left(\oplus_{i \in I} B_{i}\right)$.

It was shown in [12] that every quasi-continuous module (i.e., an extending module with $C_{3}$ condition) satisfies the full exchange property whenever it satisfies the finite exchange property. We can weaken the extending condition $\left(C_{1}\right)$ to $C_{11}$ under an additional chain condition.

Theorem 14. Let $M_{R}$ be a $C_{11}$-module which satisfies $C_{3}$ condition. If $M$ has $A C C$ on $r(m), m \in M$ then the finite exchange property of $M$ implies the full exchange property.

Proof. By [15, Lemma 4.6 (a)] and [11, Theorem 2.17] $M$ has a decomposition into indecomposable submodules. This yields the full exchange property.

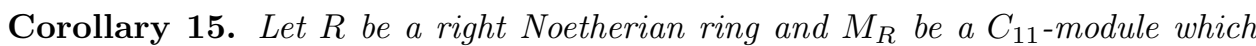
has $C_{3}$ then the finite exchange property implies the full exchange property for $M$.

Proof. It follows from Theorem 14.

\section{References}

[1] F. W. Anderson and K. R. Fuller, Rings and Categories of Modules, Springer-Verlag, 1974.

[2] D. M. Arnold and J. Hausen, A characterization of modules with the summand intersection property, Comm. Algebra, 18(1990), 519-528.

[3] G. F. Birkenmeier, F. Karabacak and A. Tercan, When is the SIP (SSP) property inherited by free modules, Acta Math. Hungar., 112(2006), 103-106.

[4] G. F. Birkenmeier, J. Y. Kim and J. K. Park, When is the CS condition hereditary?, Comm. Algebra, 27(1999), 3785-3885.

[5] N. V. Dung, D. V. Huynh, P. F. Smith and R. Wisbauer, Extending Modules, Longman, 1990.

[6] J. Hausen, Modules with the summand intersection property, Comm. Algebra, 17(1989), 135-148.

[7] I. Kaplansky, Infinite Abelian Groups, University of Michigan Press, 1969.

[8] F. Karabacak and A. Tercan,Matrix rings with the summand intersection property, Czech. Math. J., 53(2003), 621-626.

[9] F. Karabacak and A. Tercan, On modules and matrix rings with SIP-extending, Taiwanese J. Math., 11(2007), 1037-1044. 
[10] N. K. Kim and Y. Lee, Armendariz rings and reduced rings, Journal of Algebra, 223(2000), 477-488.

[11] S. H. Mohamed and B. J. Müller, Continuous and Discrete Modules, Cambridge University Press, 1990.

[12] K. Oshiro and S. T. Rizvi, Exchange property of quasi-continuous modules with the finite exchange property, Osaka J. Math., 33(1996), 217-234.

[13] S. T. Rizvi and C. S. Roman, Baer and quasi-baer modules, Comm. Algebra, 32(2004), 103-123.

[14] P. F. Smith, Modules for which every submodule has a unique closure, Ring Theory (S.Jain and S.T. Rizvi eds.) New Jersey, World Scientific, (1992), 302-317.

[15] P. F. Smith and A. Tercan, Generalizations of CS-modules, Comm. Algebra, 21(1993), 1809-1847.

[16] P. F. Smith and A. Tercan, Direct summands of modules which satisfy $\left(C_{11}\right)$, Algebra Colloq., 11(2004), 231-237.

[17] G. V. Wilson, Modules with the summand intersection property, Comm. Algebra, 14(1986), 21-38. 